\title{
A New Approach of BLDC Motor Using Unidirectional Current in the Driver Circuit and its Future Prospects
}

\author{
Komatsu Yasuhiro*, Syed Abdul Kadir Zawawi *
}

\begin{abstract}
Climate change and other pollutions make a huge demand of environment friendly and high efficient motors especially Brushless DC (BLDC) motors. Generally, bidirectional energized BLDC motors are used widely; however, inverter devices used in the driver put fear of being effected by noise. This paper proposes unidirectional energized BLDC motor which utilizes asymmetrical $H$-bridge circuit as the driver circuit. The Minato motor is one of the pioneers in unidirectional energized system. The use of bar magnets in the rotor is one of the biggest disadvantages of the motor. We proposed using tabular magnets. $T$ he paper compares the power consumption and efficiency of the Minato motor and the proposed motor. During high speed rotation, undesirable armature current is generated that has a deceleration characteristic. This current lowers the motor's efficiency. In this paper, we propose the solutions and show comparison through equations of the copper loss ratio for the Minato and our proposed motors. The third motor, which has the highest efficiency, was discovered during examination of the equations.
\end{abstract}

Keywords : BLDC motor; Minato motor, Repulsive force, Unidirectional current, Asymmetric H-bridge circuit, Back-EMF

\section{Introduction}

We are living in a world which is moved by various types of motors. There are motors which we can see vividly and also motors which are so small that they are inserted in a place we couldn't imagine that they can fit in. Among these different kinds of motors, the number of brushless motors being used is increasing rapidly in recent years due to their high efficiency and other advantages compared to the conventional motors. A unidirectional energized Brushless DC (BLDC) motor can be adopted for a small motor system. The Minato motor is one of the pioneers for unidirectional energized BLDC motor. Currently, bidirectional energized BLDC motors are being mainly studied. Since controlling the torque direction by each armature winding for a unidirectional motor system is easy, we developed a highly efficient unidirectional energized BLDC motor. In this paper, we propose a new approach of controlling unidirectional current in a small BLDC motor which we designed and the result is clearly discussed and examined [1], [3]. An asymmetric H-bridge circuit is used as a driver circuit for the motors. This circuit is hardly damaged due to malfunction, compared to the inverter used in bidirectional energized BLDC motors. Fig. 1 shows the motors' configuration, and the rotor, in which the permanent magnets are established. In the 2nd Motor, attractive and repulsive forces by permanent magnets and electromagnets are utilized. In proportion to the electromagnetic rated torque, the above fundamental unit

\footnotetext{
* Dept. of Science and Engineering, Ritsumeikan University, Japan. (komatsu@se.ritsumei.ac.jp)

Received 04 July 2011; Accepted 04 November 2011
}

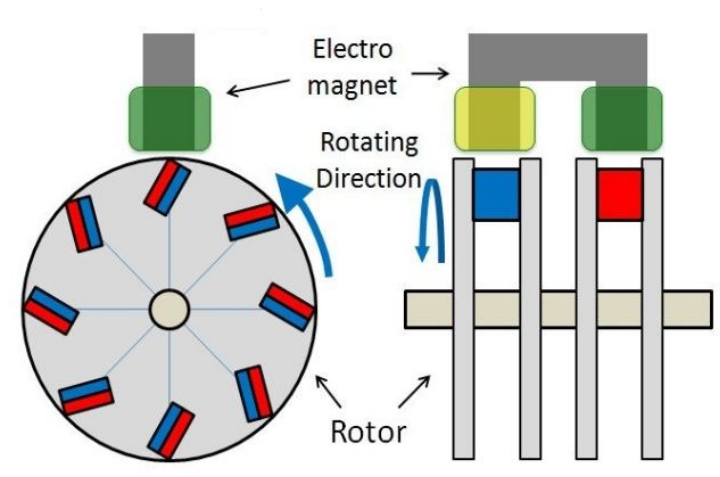

Fig 1. Configuration of proposed motors.

must be established as $\mathrm{n}$ sets. However, $\mathrm{n}$ is a natural number. The rare earth permanent magnet on the market is used in the proposed motors. Two wide planes of magnets are magnetized. The permanent magnets are fixed in the rotating disks without iron cores. Therefore, exchanging the demagnetized magnet and recycling are easy. The decision in the current period, in which the current flows in the electromagnet, greatly influences the characteristic of the motors. The period in which the current flows is called the 'on-period' and the period that current does not flow is the 'off-period.' Although the "2nd motor" gets better efficiency compared to the Minato motor, it uses high current value which can easily damaged the converter device inside the driver circuit. From the experiment, we found out that by using the same method to drive Minato motor, we could achieve higher efficient using our proposed design; hence, we called it the "3rd motor". We propose a new approach of controlling unidirectional current in the 
winding to achieve higher efficient in comparison to the Minato motor.

\section{The Minato motor}

\subsection{Structure and principle of the Minato motor}

In Fig. 2, the structure and principle of the Minato motor is shown [2]-[6]. The rotor consists of permanent magnets without an iron core. The stator consists of electromagnets that include iron cores. Multiple electromagnets can be established and linked with parallel and series connections. The permanent magnet of the rotor is long, and its pole area is small. It is called a bar magnet.

From Fig. 2, the electromagnet's center-axis is not facing the radial. If the normal direction is the electromagnet's direction, the rotor's rotation direction will be the opposite. In the Minato motor, strong repulsive force is only used when the permanent magnets and the electromagnets are adjacent. Fig. 2 shows that the attractive force between the $\mathrm{N}$ and $\mathrm{S}$ poles is very small. In this motor, the on-period is short. By generating a strong repulsive force, demagnetization of the permanent magnet can be generated. However, demagnetizing the bar magnet is difficult. A bar magnet is adopted for this desirable quality. In the on-period, since the magnetic flux linkage of the armature winding is big due to the magnetic flux of the permanent magnets, the back-EMF in the armature winding is big. In the off-period, a back-EMF is not utilized. The back-EMF constant in the on-period is defined by the backEMF per-rotation speed, and equals the torque constant, which is defined by the torque value per-armature current.

In Fig. 2, if the switch is closed in a short period, the rotor rotates. At that time, a large repulsive force is generated from the $\mathrm{N}$ pole, which rises. When the next permanent magnet is adjacent to the electromagnet, a similar action is repeated, and the rotor keeps its rotation. In the real machine, a large repulsive force, which rises by the $\mathrm{S}$ poles of the permanent magnet and the electromagnet, is also utilized.

\subsection{Details of the Minato motor}

In the Minato motor, only strong repulsive force is used, in which a permanent magnet is adjacent to the electromagnet. When the current is 0 , the electromagnet and permanent magnet most closely approach each other. To narrow the gap, the magnetic pole face must be small. Therefore, a bar magnet is used, in which the magnetic pole's area is small. The on-period is greatly shortened. Fig. 3 shows the single phase part of the drive circuit, which is called a 'three phase asymmetrical $\mathrm{H}$ bridge circuit' that is used to drive the Switched Reactance Motor (SRM). This drive circuit is utilized in the Minato motor.

The rotational position sensing circle (Fig. 4) is used to control the switch SW1 and SW2 of the drive circuit (Fig. 3 ). The operation that decides whether to apply the voltage is carried out by a photo-interrupter and a rotational position sensing disc, where the light shielding and transparent regions are established. The rotational position sensing disc of the Minato motor is shown in Fig. 4.

From Fig. 4, on the clear side, light can flow through the photo-interrupter, and switches SW1 and SW2 are closed. During that time, the motor rotates until the dark side appears. We define the clear side $\left(15^{\circ}\right)$ as the "on-region" and the dark side as the "off-region". The on-region is small. Therefore, the on-period of the switching period, Ton/T is small.

\subsection{Measurement of torque constant and back-EMF constant}

The torque constant is the electromagnetic torque per unit current. The back-EMF constant is the back-EMF perunit rotational speed. The torque and back-EMF constants for multiple conductors like motors are obtained using the following equations expressed below.

The linear conductor of length $l$ exists in the uniform magnetic flux density $B$. The magnetic flux and the conductor are orthogonalized. Force $\mathrm{f}$ in the conductor is shown by the following equation, when current $i$ is flowing in the conductor:

$$
f=B l i
$$

Next, the torque in the motor's conductor is examined. It is assumed that the magnetic flux density on the straight line in the column surface is the same. The magnetic flux density component which is perpendicular to the column surface is assumed as B. This magnetic flux distribution is called the cylindrical magnetic flux. Force $f$ of the tangential direction component that works in the conductor, can also be shown by Eq. (1), when current $i$ is flowing in a straight line conductor of length 1 on the column surface. Torque $\mathrm{T}$, which works in the conductor, can be shown by the following equation, if the column radius is assumed as r:-

$$
T=B l i r
$$

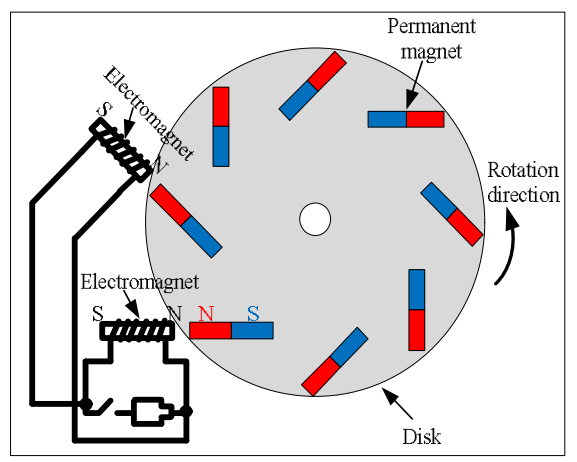

Fig 2. Structure and principle of the Minato motor using strong repulsive force when permanent magnets closely approach the electromagnets. 


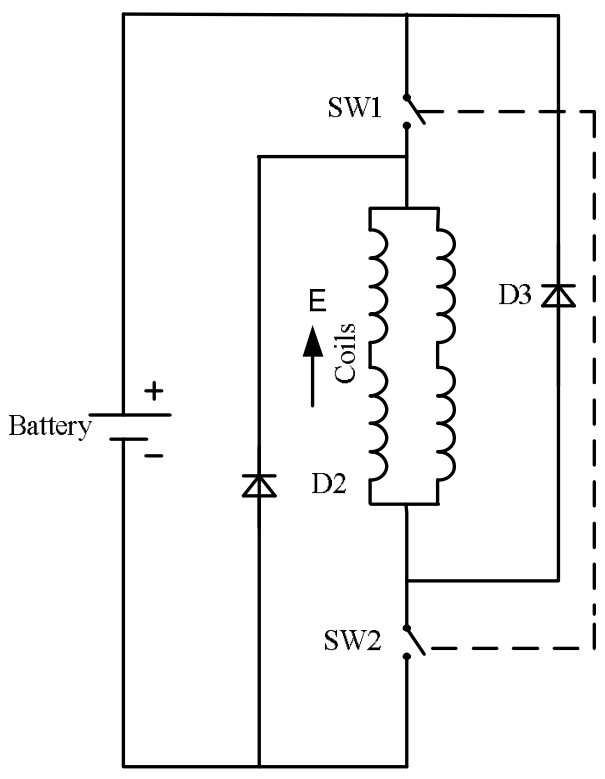

Fig 3. Drive circuit of Minato motor.

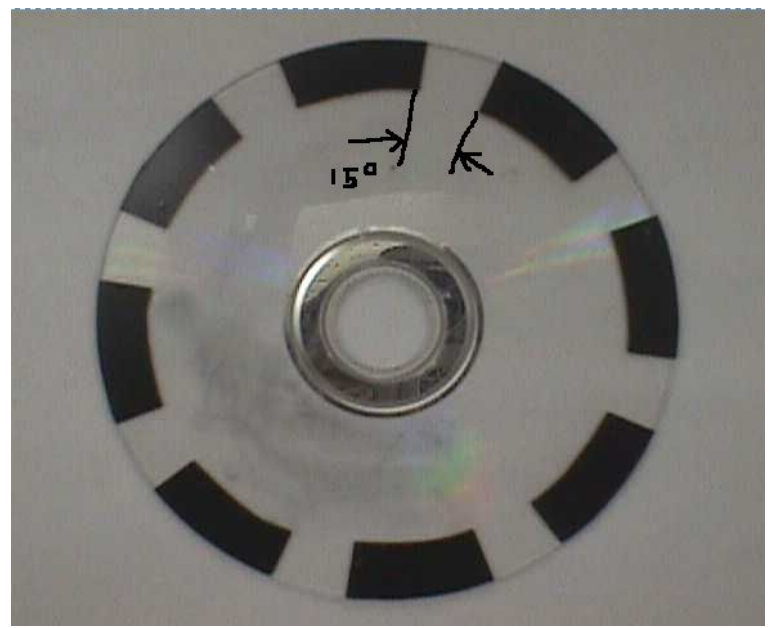

Fig 4. Rotational position sensing disc of the Minato motor when on-region is $15^{\circ}$.

We assume that the magnetic flux is made by rotating the permanent magnet. In the pole face of the permanent magnet, reverse force $f$ ' occurs due to Newton's 3rd Law (Action and Reaction) at the equal force.

Torque $T^{\prime}$ is generated by $f^{\prime}$ in the pole face of the permanent magnet. $T$ and $T$ ' have the same magnitude, but their direction is reversed, which can be proven by using the law of sines.

The $q$ pieces of the above conductors exist and are connected in series. Each conductor has different $B$ and $r$ values, and different positions. Let current $i$ and length $l$ for each conductor be the same. In that case, the following equation can be written:-

$$
T=\sum_{j=1}^{q} B_{j}(\theta) r_{j} l i
$$

is a rotational angle of the rotor. The following equation is obtained from Eq. (3):-

$$
\frac{T}{i}=\sum_{j=1}^{q} B_{j}(\theta) r_{j} l
$$

$T / i$ is the torque constant.

Next, the examination for back-EMF can be expressed as below.

The back-EMF of the conductor, $e$ can be shown as follows at the cylindrical magnetic flux, when the conductor of length $l$ moves in the circular motion of radius $r$ and angular velocity $\omega:-$

$$
e=B l \omega r
$$

Equation (5) is also formed, even during the conductor rests, and the magnetic field moves in a circular motion. $q$ pieces of the above conductor exist and are connected in a series. Each conductor has different $B$ and $r$ values. Let angular velocity $\omega$ and length $l$ for each conductor be the same. In that case, the following equation can be written:-

$$
e=\sum_{j=1}^{q} B_{j}(\theta) r_{j} l \omega
$$

$\theta$ is a rotational angle of the rotor. The following equation is obtained from Eq. (6):-

$$
\frac{e}{\omega}=\sum_{j=1}^{q} B_{j}(\theta) r_{j} l
$$

$e / \omega$ is the back-EMF constant. From Eq. (4) and (7), the torque constant becomes equal to the back-EMF constant

\subsection{Disadvantage of the Minato motor}

If the electromagnetic current increases greatly, a large repulsive force is created. In this case, demagnetization of the permanent magnet can be generated. Therefore, the amplitude of the repulsive electromagnetic torque should be limited.

In low speed operation, the off-period is long. If there is a large load torque in the off-period, the deceleration quantity increases. The speed can become 0 before the photo-interrupter reaches the on-region. Therefore, large torque operation at low speed with the Minato motor is difficult. When this motor is used in electric cars, starting it will be difficult.

In Fig. 5, the electromagnet current waveforms in the low and high speed rotations of the Minato motor are shown. 


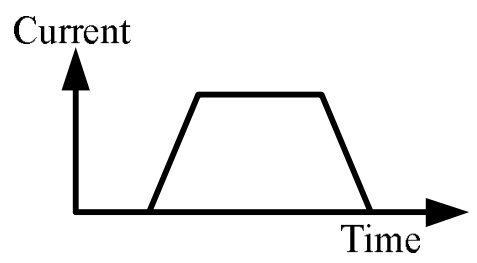

a. Electromagnet current waveform in the low-speed rotation

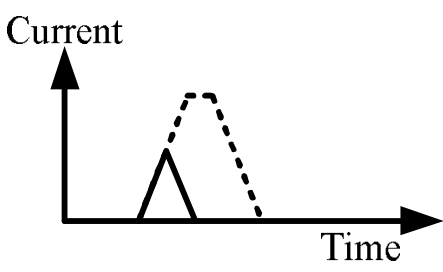

b. Electromagnet current waveform in the high-speed rotation

Fig 5. Electromagnet current waveforms in low and high speed rotations of the Minato motor.

Fig. 5(a) shows the electromagnet current waveform in low-speed rotation whereas Fig. 5(b) shows it in highspeed rotation. Since the on-period is generally short in the Minato motor, the on-period in the high speed rota tion can be further shortened. The current decrease begin $\mathrm{s}$ from the middle point of the current increase, when it is in the extreme mode. Therefore, the electromagnet average current is small in the high-speed rotation. The average torque is small, because the current is proportional to the torque.

Even though the armature current is small, a large applied voltage is required since the back-EMF is large. The product of the current and the voltage, in other words the electric power, does not decrease. Therefore, the Minato motor does not save energy.

\section{Features and advantages of the proposed Motors}

The proposed motors, which belong to the unidirectional energized BLDC motor, are shown in Fig. 6. They resemble the configuration of the Minato motor but differ in magnet type.

The fundamental configuration of the " $2^{\text {nd" }}$ and Minato motors is almost same. In the " $2^{\text {nd }}$ motor", an on-pattern is adopted, in which generating demagnetization is difficult. The off-period of the Minato motor becomes approximately the " 2 nd motor's on-period. Therefore, the on-period of the " $2^{\text {nd }}$ motor" is longer, and its off-period is shorter. In addition, large torque operation at low speed becomes

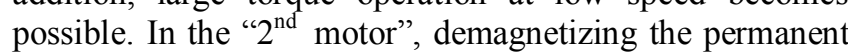
magnets is difficult since both weak attractive and repulsive forces are utilized.

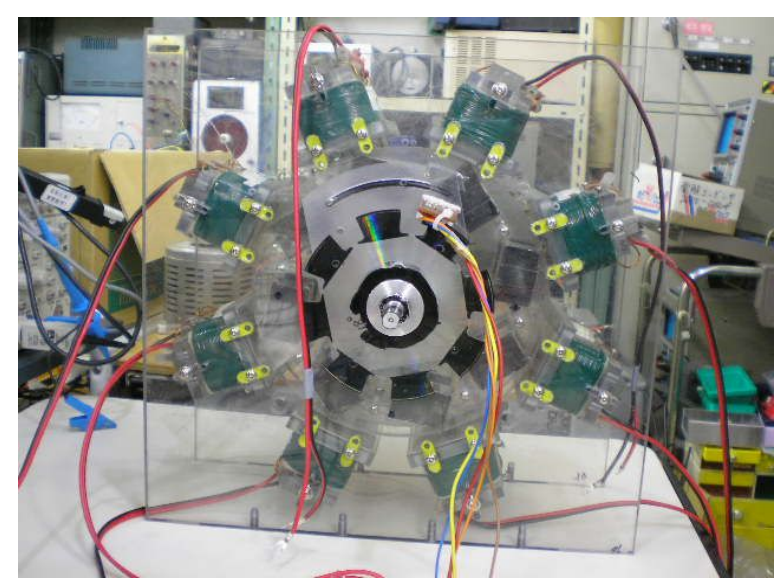

Fig 6. Appearance of the proposed motor.

A permanent magnet, whose length is short and whose magnetic pole area is big, can be used (a tabular magnet). This magnetic flux quantity, which comes out from the magnetic face, is big. Therefore, the a $\mathrm{ve} \mathrm{r}$ a $\mathrm{g} \mathrm{e}$ electromagnetic torque increases. The rectangular wave voltage can also be applied to the electromagnets for the "2nd motor". In high speed rotation, the on-periods of both motors are shortened. In the case of the Minato motor, since its on-period is short, when the rotational speed increases, the current decrease may start from the middle point of the current increase. Therefore, large torque cannot be generated.

For the " $2^{\text {nd }}$ motor", the same thing occurs when the rotational speed increases. However, the effect of current increase and decrease during rotation is lower than in the Minato motor. Hence, the proposed motor is more suitable for high speed rotation.

We assumed that the armature currents of both motors are rectangular wave. The cases for a) the same electromagnets and the same input power, and b) the same rotational speed and the same average generated torque are considered in both motors. The amplitude of the rectangular wave current for the Minato motor is big and, in that case, the on-period is short. The situation of the "2nd motor" is the opposite.

In Table 1, the comparisons of the copper loss ratio for the two motors are shown. Since the input and output are same in both motors, $m^{2} k$ must be equal to 1 . By using Table 1, the calculation of per-unit (PU) resistance for the Minato motor and the " $2^{\text {nd }}$ motor" can be derived and is shown in Table 2. From the calculation, PU resistance for Minato motor is approximately near the copper loss ratio as shown in Table 1. Similar calculations can also be made for the " $2^{\text {nd }}$ motor". These calculation results are more appropriate.

In the " $2^{\text {nd }}$ motor", the value of $k$ exceeds 1 , but the value of $\mathrm{m}$ is smaller than 1 . If $1 / \mathrm{m}^{2} k$ is smaller than 1 , the " $2^{\text {nd }}$ motor" outperforms the Minato motor. Since the onperiod of the " $2^{\text {nd }}$ motor" is big, $k$ becomes bigger, reducing the value of $1 / \mathrm{m}^{2} k$. Therefore, the " $2^{\text {nd }}$ motor" is better than the Minato motor. 
Table 1. Comparison of various quantities between the Minato and the " $2^{\text {nd }}$ Motor"

\begin{tabular}{|c|c|c|}
\hline & Minato motor & The " $2^{\text {nd }}$ Motor" \\
\hline $\begin{array}{l}\text { Torque constant per } \\
\text { unit electromagnetic } \\
\text { during on-period }\end{array}$ & $k_{T}$ & $m k_{T}$ \\
\hline On-period & $T_{\text {on }}$ & $\kappa T_{\text {on }}$ \\
\hline Period & $T$ & $T$ \\
\hline $\begin{array}{l}\text { Number of } \\
\text { electromagnets }\end{array}$ & $n$ & $n$ \\
\hline Parallel circuit number & $a_{m}$ & $a_{p}$ \\
\hline $\begin{array}{c}\text { Series connected } \\
\text { electromagnets } \\
\text { number }\end{array}$ & $\frac{n}{a_{m}}$ & $\frac{n}{a_{p}}$ \\
\hline Total peak current & $I_{m}$ & $I$ \\
\hline $\begin{array}{l}\text { Peak current per-unit } \\
\text { electromagnet }\end{array}$ & $\frac{I_{m}}{a_{m}}$ & $\frac{I}{a_{p}}$ \\
\hline $\begin{array}{c}\text { Peak torque } \\
\text { per-unit electromagnet }\end{array}$ & $k_{T} \frac{I_{m}}{a_{n}}$ & $\frac{m k_{T} I}{a_{p}}$ \\
\hline Total peak torque & $n k_{T} \frac{I_{m}}{a_{n}}$ & $n k_{T} \frac{m I}{a_{p}}$ \\
\hline Total average torque & $n k_{T} \frac{I_{m}}{a_{m}} \times \frac{T_{o n}}{T}$ & $n k_{T} \frac{m I}{a_{p}} \times \frac{T_{o n}}{T} \times \boldsymbol{K}$ \\
\hline $\begin{array}{l}\text { Condition of equality } \\
\text { of average torque }\end{array}$ & $\begin{aligned} n k_{T} \frac{I_{m}}{a_{m}} \times \frac{T_{o n}}{T} & = \\
I & =\end{aligned}$ & $\begin{array}{l}=n k_{T} \frac{m I}{a_{p}} \times \frac{T_{o n}}{T} \times \boldsymbol{\kappa} \\
\frac{a_{p} I m}{m a_{m 3} \kappa}\end{array}$ \\
\hline $\begin{array}{l}\text { Back-EMF at on- } \\
\text { period per-unit } \\
\text { electromagnet }\end{array}$ & $U$ & $m U$ \\
\hline Total back-EMF & $\frac{n}{a_{m}} U$ & $\frac{m n}{a_{p}} U$ \\
\hline $\begin{array}{l}\text { Confirmation for the } \\
\text { equality of Input } \\
\text { energy during } \\
\text { on-period }\end{array}$ & $\begin{aligned} & \frac{n}{a_{m}} U I_{m} \times T_{o n} \\
= & \frac{n U I_{m} T_{o n}}{a_{m}}\end{aligned}$ & $\begin{array}{c}\frac{m n}{a_{p}} U I \times \kappa T_{o n} \\
=\frac{n U I_{m} T_{o n}}{a_{m}}\end{array}$ \\
\hline Input average power & $\frac{n U I_{m} T_{o n}}{a_{m} T}$ & $\frac{n U I_{m} T_{o n}}{a_{m} T}$ \\
\hline Current effective value & $\begin{aligned} & \sqrt{\frac{1}{T} \int_{0}^{T_{o n}} I_{m}^{2} d t} \\
= & I_{m} \sqrt{\frac{T_{o n}}{T}}\end{aligned}$ & $\begin{aligned} & \sqrt{\frac{1}{T} \int_{0}^{\kappa T_{o n}} I^{2} d t} \\
= & \frac{a_{p} I_{m}}{m a_{m} \kappa} \sqrt{\frac{\kappa T_{o n}}{T}}\end{aligned}$ \\
\hline $\begin{array}{c}\text { Equivalent resistance } \\
\text { (r r resistance } \\
\text { per-unit } \\
\text { electromagnet) }\end{array}$ & $\frac{n}{a_{m}^{2}} r$ & $\frac{n}{a_{p}^{2}} r$ \\
\hline Total copper loss & $\begin{array}{l}\frac{n r}{a_{m}^{2}}\left(\operatorname{Im} \sqrt{\frac{T_{o n}}{T}}\right)^{2} \\
=\frac{n r}{a_{m}^{2}} \operatorname{lm}^{2} \frac{T_{o n}}{T}\end{array}$ & $\begin{array}{l}\frac{n r}{a_{p}}\left(\frac{a_{p} I_{m}}{m a_{m} \kappa} \sqrt{\frac{\kappa T_{o n}}{T}}\right)^{2} \\
=\frac{n r}{a_{m}^{2} m^{2} \kappa} I_{m}{ }^{2} \frac{T_{o n}}{T}\end{array}$ \\
\hline$\frac{\text { Total copper loss }}{\text { Input average power }}$ & $\frac{r I_{m}}{a_{m} U}=\frac{\frac{r}{U}}{\left(\frac{I_{m}}{a_{m}}\right)} \equiv r_{p}$ & $\frac{r I_{m}}{a_{m} U m^{2} \kappa}=\frac{\frac{r}{m U}}{\left(\frac{I}{a_{p}}\right)} \equiv \frac{1}{m^{2} \kappa} r_{p}$ \\
\hline
\end{tabular}

Table 2. Calculations of per-unit resistance for the Minato motor and the " $2^{\text {nd }}$ Motor"

\begin{tabular}{|c|c|}
\hline Minato motor & The $2^{\text {nd }}$ Motor \\
\hline $\mathrm{r}_{\mathrm{pu}}=\frac{\frac{\text { Equivalent Resistance }}{\text { Rated terminal voltage }}}{\text { Rated terminal current }}$ & $\mathrm{r}_{\mathrm{pu}}=\frac{\text { Equivalent Resistance }}{\text { Rated terminal voltage }}$ \\
\hline$\approx \frac{\text { Equivalent Resistance }}{\frac{\text { Rated back - EMF }}{\text { Rated terminal current }}}$ & $\approx \frac{\text { Equivalent Resistance }}{\frac{\text { Rated back - EMF }}{\text { Rated terminal current }}}$ \\
\hline$\approx \frac{\frac{n r}{a_{m}^{2}}}{\frac{\left(\frac{n}{a_{m}}\right) U}{I_{m}}}$ & $\approx \frac{\frac{\mathrm{nr}}{\mathrm{a}_{\mathrm{p}}{ }^{2}}}{\frac{\left(\frac{\mathrm{mn}}{\mathrm{a}_{\mathrm{p}}}\right) \mathrm{U}}{\mathrm{I}}}$ \\
\hline$=\frac{n r a}{a_{m} I_{m}}$ & $=\frac{n r a_{p} I}{a_{p}{ }^{2} m n U}$ \\
\hline$=\frac{\mathrm{rI}_{\mathrm{m}}}{\mathrm{a}_{\mathrm{m}} \mathrm{U}}$ & $=\frac{\mathrm{rI}}{\mathrm{a}_{\mathrm{p}} \mathrm{mU}}$ \\
\hline
\end{tabular}

The motor, which has small copper loss per-unit input power, has high efficiency. In other words, a motor with high values of $k$ and $m$, has high efficiency. Therefore, tabular magnets should be used for a bigger value of $k$. Furthermore, when both magnets approach closely, the flow of the electromagnetic current should be controlled to attain bigger value of $m$. The aforementioned facts explain why the Minato motor should use tabular magnets instead of bar magnets. We call this improved Minato motor the " 3 rd motor". By placing tabular magnets in the rotor, a reciprocal attractive force which is generated between the electromagnets makes demagnetization difficult. This characteristic is one of the advantages of the proposed motors.

\section{Motor driver circuit for proposed motors}

Since the proposed motors consist of a single phase winding, each armature winding can be connected in serial and parallel. Fig. 7 shows that SW1 and SW2 are simultaneously turned on and off. The coil's magnetic energy can be regenerated in Fig. 7(a). In that case, a storage battery or rectifier circuits are used as a DC power source.

The storage battery deteriorates when the regeneration current of a frequency larger than the commercial frequency flows into it. In general, negative current cannot 


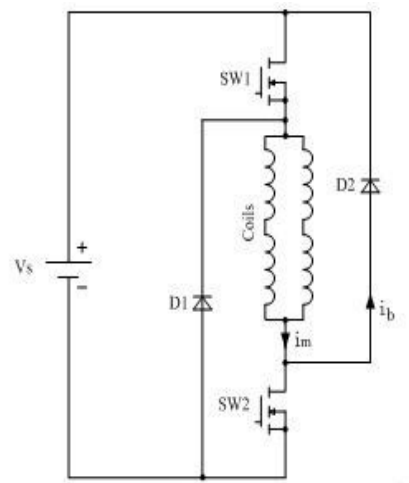

(a)

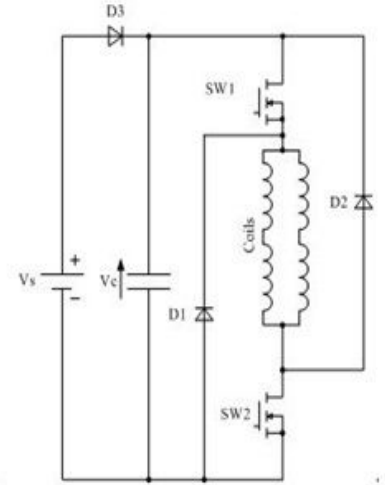

(b) (a) Circuit in which regeneration current flows in DC power supply

(b) Circuit in which regeneration current flows in $\mathrm{c}$ apacitor.

Fig. 7. Details of motor driver circuit

flow in the rectifier circuits. To avoid running the regeneration current in the DC power source, the circuit of Fig. 7(b) is adopted.

\section{Motor-Generator as an application for the proposed motors}

A permanent magnet synchronous generator is made by placing the windings for the power generation in the space between each motor winding [7]. The following are the experimental results of the proposed motor-generator (M$\mathrm{G})$. The value of the electromagnets equals 8 and the rotor diameter is $20[\mathrm{~cm}]$. Four sets of electromagnets are used for the motor, and the other four sets of electromagnets are used for the generator. The source voltage was DC60[V], and the motor current was DC1.6[A]. Therefore, the input power was $42.24[\mathrm{~W}]$. The power of the generator is $32[\mathrm{~W}]$, and the combined efficiency of the motor-generator is $77.51[\%]$.

Table 3 compares the efficiency of the Minato Motor and the proposed motors in terms of M-G. The " 3 rd motor's" efficiency is clarified as the highest. These M-Gs can also be used as inverters for the system interconnections. Equipment under $3[\mathrm{~kW}]$ is desirable.

\section{Generation of induction brake current and Prevention method}

Undesired induced current can flow in the armature winding (coil) of the proposed motors (the "2nd motor" during its off-period and the "3rd motor" during its onperiod). In this paper, the current is called 'induction brake
Table 3. Comparison of highest efficiency between The Minato motor and the proposed motors

\begin{tabular}{|c|c|c|}
\hline & Clear Region & $\begin{array}{c}\text { Highest } \\
\text { Efficiency [\%] }\end{array}$ \\
\hline Minato Motor & $15^{\circ}$ & 56.87 \\
\hline The 2nd Motor & $21^{\circ}$ & 75.69 \\
\hline The 3rd Motor & $18^{\circ}$ & 77.51 \\
\hline
\end{tabular}

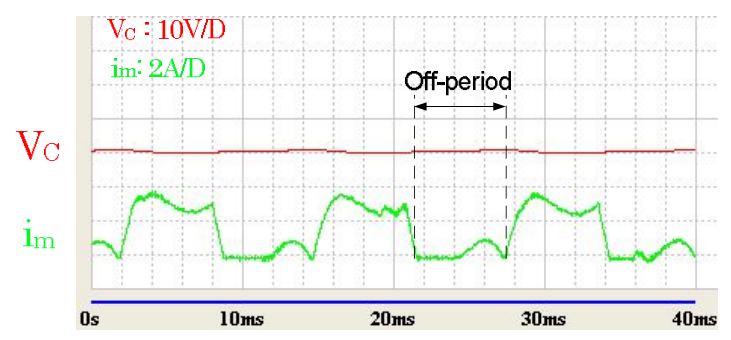

Fig 8. im, Vc waveform when $\mathrm{C}$ is $4810[\mu \mathrm{F}]$ (Vs: 20 [V], rotational speed: $588.2[\mathrm{rpm}]$ )

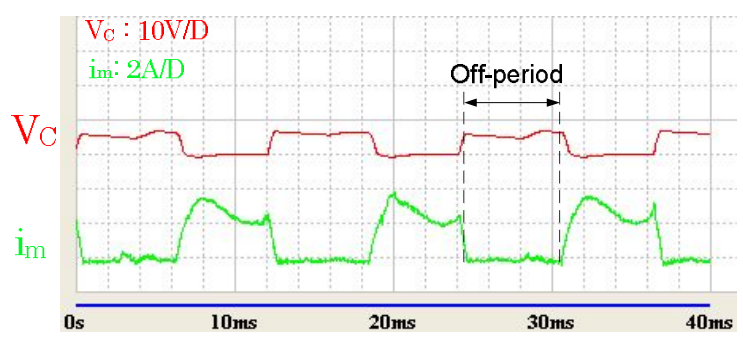

Fig 9. im, Vc waveform when $\mathrm{C}$ is $110[\mu \mathrm{F}]$ (Vs: 20 [V], rotational speed: $616.2[\mathrm{rpm}]$ )

current', iB. During the off-period, if the rotating speed is high, back- EMF becomes the negative of a high absolute value. $\mathrm{iB}$ is generated when the absolute value of backEMF is higher than the absolute value of Vc. iB makes the deceleration torque component TB. Consider $\omega$ the rotating angular velocity. The electric power which is reduced by the copper loss due to iB from Eq. $\omega \mathrm{TB}$, is accumulated in the capacitor. Capacitor voltage $\mathrm{Vc}$ becomes roughly DC power source voltage $\mathrm{Vs}$, when electric capacitance $\mathrm{C}$ of the capacitor is big. The value of $\mathrm{C}$ should be increased, if the rated voltage of the FET is considered. The motor decelerates due to $\mathrm{iB}$, and $\mathrm{iB}$ lowers the efficiency. Therefore, the generation of $\mathrm{iB}$ should be suppressed. $\mathrm{iB}$ is generated when the Vc in the off-period is smaller than the induced EMF. Armature winding terminal current im wave, etc. are shown in Fig. 8 where im in the off-period is iB.

Fig. 9 shows im wave, etc. when $110[\mu \mathrm{F}]$ of capacitor is used. The results shown in both figures are conducted with the "2nd motor". In the off-period, Vc increases approximately $5.6[\mathrm{~V}]$ and $\mathrm{iB}$ is approximately 0 . Therefore, the generation of $\mathrm{iB}$ can be prevented by decreasing the capacitance value in the motor drive circuit.

The integral shape of the motor generator was constructed inside the proposed motor where the AC generator winding was prepared. Table 4 shows the overall 
efficiency $\eta$ of the motor- generator. The rotation speed of the motor-generator can be increased by lowering the capacitance value of the capacitor. Nevertheless, the motorgenerator's $n$ also increases.

\section{Experiment results}

The constructional element of the proposed motor is mainly plastic. Therefore, the rotor is light and has small core loss. The consumed electric power of an electric fan on the market using a single phase induction motor (the rated power $147[\mathrm{~W}]$ ) that has distributed winding is 96.7 [W] and the rotational speed is set at 1200 [rpm]. After this, in the case of equal operation, the consumed electric power of the proposed motor and various types of motors on the market were measured. In the proposed motor, the electromagnet's number is four and rotor diameter is $10[\mathrm{~cm}]$. The dimension of each tabular magnet is 1 [in] $\times 1[\mathrm{in}] \times 1[\mathrm{~cm}]$ in the proposed motors. The measurement result is shown at Table 5. From the table, the power consumption for the proposed motor $\left(3^{\text {rd }}\right.$ Motor) is the lowest.

Table 4. The change of overall efficiency motor-generator due to the value change of $C$

\begin{tabular}{|c|c|c|c|c|c|}
\hline$V S$ & $C$ & $\begin{array}{c}\text { Average } \\
\text { value of } \\
i_{m}\end{array}$ & $\begin{array}{c}\text { Average } \\
\text { value of } \\
V c\end{array}$ & $\begin{array}{c}\text { Rotating } \\
\text { Speed }\end{array}$ & $\begin{array}{c}\text { Overall } \\
\text { Efficiency } \\
\eta\end{array}$ \\
\hline$[\mathrm{V}]$ & {$[\mu F]$} & {$[\mathrm{A}]$} & {$[\mathrm{V}]$} & {$[\mathrm{rpm}]$} & {$[\%]$} \\
\hline \multirow{2}{*}{20} & 4810 & 1.18 & 19.0 & 588.2 & 67.11 \\
\cline { 2 - 6 } & 110 & 1.24 & 21.8 & 616.2 & 69.39 \\
\hline \multirow{2}{*}{25} & 4810 & 1.29 & 24.0 & 722.5 & 70.2 \\
\cline { 2 - 6 } & 110 & 1.36 & 27.4 & 757.6 & 72.08 \\
\hline
\end{tabular}

Table 5. The measurement result of power consumption of various motors at $1200[\mathrm{rpm}]$

\begin{tabular}{|c|c|c|c|c|}
\hline $\begin{array}{c}\text { Motor's } \\
\text { type }\end{array}$ & $\begin{array}{c}\text { Voltage } \\
{[\mathrm{V}]}\end{array}$ & $\begin{array}{c}\text { Current } \\
{[\mathrm{A}]}\end{array}$ & $\begin{array}{c}\text { Power } \\
\text { consumpti } \\
\text { on [W] }\end{array}$ & Rating \\
\hline $\begin{array}{c}\text { Proposed M } \\
\text { otor }\end{array}$ & 45.93 & 0.975 & 44.5 & $\sim$ \\
\hline $\begin{array}{c}\text { BLDC } \\
\text { Motor }\end{array}$ & 24.0 & 2.10 & 50.0 & $100[\mathrm{~W}]$ \\
\hline $\begin{array}{c}\text { DC Servo- } \\
\text { Motor }\end{array}$ & 36.24 & 1.44 & 51.8 & $90[\mathrm{~W}]$ \\
\hline \begin{tabular}{c} 
DC Motor \\
\hline Induction \\
Motor
\end{tabular} & 16.91 & 3.42 & 57.5 & $24[\mathrm{~V}]$ \\
\hline
\end{tabular}

\section{Conclusion}

High efficient single phase unidirectional energized BLDC motors are developed in order to improvise Minato motor's disadvantage. In other words, these are the " 2 nd motor" and the the " $3^{\text {rd }}$ motor". The result of this research is shown below.

1. In the Minato motor, only the strong repulsive force is utilized when the permanent magnets and the electromagnets are adjacent.

2. In the Minato motor, since the permanent magnet can be demagnetized, a bar magnet is used to prevent this.

3. In the Minato motor, the armature current is small. However, the applied voltage is high. Therefore, the power input is not necessarily small. Since the onperiod is small, the copper loss increases; lowering the efficiency.

4. In the Minato motor, the off-period is long. In that period, if large load torque occurs, the deceleration quantity increases and the motor may stop. Therefore, low speed and large torque operation becomes difficult.

5. In the " $2^{\text {nd }}$ motor", the on-period of the Minato motor resembles the off-period. Therefore, attractive and repulsive forces are utilized at the interval when the permanent magnet and the electromagnets separate. Hence, demagnetization becomes difficult.

6 . In the " 2 nd motor", a tabular magnet can be utilized since demagnetization is difficult.

7. In the proposed motors, low speed and large torque operation is possible because the off-period is short.

8. The induction break current of the proposed motors can be controlled due to the decrease of the motor driver circuit's capacitance. Hence, the motor's efficiency can be increased.

9. In the " 3 rd motor", where the on-period is long and the magnetic poles of the permanent magnet and electromagnet approach each other during the onperiod, the copper loss per-unit input power decreases. The efficiency of the " 3 rd motor" is higher than the " 2 nd $m o t o r "$.

\section{Acknowledgements}

The authors would like to thank the technical staff of Ritsumeikan University Workshop Laboratory, Mr. Toshio Yumiyama, Mr. Hajime Yamaue, and Mr. Hiroyuki Sugimoto for making the motor parts.

\section{References}

[1] Komatsu Yasuhiro et al, Magnetic Rotating Apparatus, Japan Patent No. 3897043, January 2007. 
[2] Minato Kohei, Magnetic Rotating Apparatus, United States Patent No.4751486, June 14, 1988.

[3] Yasuhiro Komatsu, Tur-Amgalan Amarsanaa, and Yoshihiko Araki, "Highly Effective Brushless DC Motor in Which the Unidirectional Current Flow", ICEMS2009 No. DS2G1-14

[4] Minato, Kohei. “Magnetic Rotating Apparatus," United States Patent No. 5594289. Jan. 14, 1997

[5] Minato, Kohei and Minato, Nobue. "Direct-Driven Magnetic Rotating Apparatus," United States Patent No. 7075200 B2. July 11,2006

[6] Minato, K and Minato, N. "Magnetic Rotating Motor Generator," United States Patent No. 7148596 B2. Dec 12, 2006

[7] Komatsu Yasuhiro et al, "Unidirectional Energized BLDC Motor with AC Output Winding and the Motor System," Japan Patent No.4569883, August 2010.

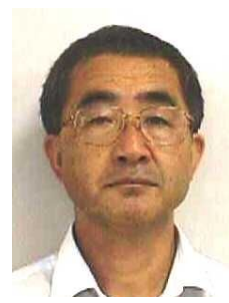

Komatsu Yasuhiro received Ph.D. degree in electrical engineering from Kyoto University in 1991. His research interests are power electronics and electric machines.

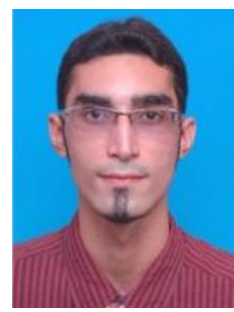

Syed Abdul Kadir Zawawi received B.S and M.S degrees in electrical engineering from Ritsumeikan University. His research interests are power electronics and electric machines. 